\title{
The Newest Frontier in Cholesterol Management; PSCK 9
}

\author{
Alexander Dashwood* and Jayasinghe $\mathbf{R}$
}

Gold Coast University Hospital, Queensland, Australia

\begin{abstract}
Cardiovascular disease from atherosclerosis remains a major cause of death in Australia. Epidemiological studies have demonstrated that reducing low-density lipoprotein cholesterol (LDL-C) strongly correlates with improved cardiovascular risk in. Although statin based therapy remains our most effective and first line therapy, certain cohorts remain refractory to their benefits. Proprotein convertase subtilisin/kexin type 9 (PCSK9), a monoclonal antibody, is currently under research as a novel non-statin based therapy in multiple clinical trials. PCSK9 targets low-density lipoprotein receptors for internalisation and lysomal degradation. This affects cholesterol homeostasis by reducing the cells ability to internalise circulating apoliprotein B. Early trials have shown clear benefits in LDL-C reduction without major adverse incidence in populations intolerant to statins, those with familial hypercholesterolaemia and patients requiring additional treatment to meet cholesterol targets despite statin based therapy. This article is a clinical focus reviewing the current phase I, II and III trials being conducted and the potential benefits a monoclonal antibody to PCSK9 may hold for cholesterol regulation in the future.
\end{abstract}

Keywords: Hypercholesterolemia; LDL cholesterol; Coronary vascular disease; PCSK9

\section{Background}

It has now emerged that by blocking low-density lipoprotein receptor degradation by proprotein convertase subtilisin/kexin type 9, low-density lipoprotein cholesterol (LDL-C) can be reduced very effectively and significantly. These monoclonal antibodies are likely to provide a robust and reasonable alternative for certain populations; those intolerant to statins, those who do not attain recommended LDL-C levels despite maximal therapy and finally individuals with Familial Hypercholesterolemia.

\section{Introduction}

Cardiovascular disease, developing from atherosclerosis, continues to be a leading cause of death in industrialized countries. Control of cholesterol and specifically reduction of low-density lipoprotein cholesterol (LDL-C) has been demonstrated to strongly correlate with improved cardiovascular risk in epidemiological studies. Subsequently, all clinical guidelines have adopted specific LDL-C goals based on cardiovascular outcome trials [1]. In Australia, high-risk individuals for coronary vascular disease (CVD) and those with pre-existing CVD have a target LDL-C of less than $2.5 \mathrm{mmol} / \mathrm{L}$ and $1.8 \mathrm{mmol} / \mathrm{L}$ respectively. First line therapy remains statins; hydroxyl-methylglutaryl co-enzyme A reductase inhibitors. They are currently recognized as our most effective agent with up to $63 \%$ reduction in LDL-C from baseline [2]. However, despite their success, significant gaps remain in the treatment of hypercholesterolemia [3]. 10-20\% of patients are unable to tolerate statins due to muscle related side effects and therefore do not achieve recommended LDL-C target levels. Other individuals continue to experience complications of atherosclerosis despite being on an adequate regime. Unfortunately our second line therapies; ezetimibe, fibrates and bile acid sequestrates have shown only limited clinical benefit. Ezetimibe, the most favoured second line treatment, reduces LDL-C levels by up to $18 \%$, which is unlikely to meet the LDL-C goals our guidelines strive for unless used as dual treatment with statins. For these populations an alternative non-statin cholesterol agent is required. It has now emerged that by blocking the degradation of the low-density lipoprotein receptor (LDLR) by proprotein convertase subtilisin/kexin type 9 (PCSK9), LDL-C can be reduced very effectively and significantly.

\section{What is PCSK9?}

Central to cellular cholesterol homeostasis is the hepatic LDLR. PCSK9 plays a pivotal role in regulating LDLR recycling which is fundamental to the hepatocyte cells ability to efficiently internalize circulating apoliprotein B (Apo B) [4]. LDLR binds Apo B resulting in internalization of the complex, allowing Apo B to undergo lysosomal degradation and release of cholesterol. The free LDLR is then recycled back to the hepatocyte membrane to once again bind circulating Apo B [5]. PCSK9, secreted by hepatocytes, binds the LDLR and limits their surface expression by targeting them for lysosomal degradation following internalization into the cell (Figure 1). In 2003 Abifadel et al mapped a mutation to chromosomal locus $1 \mathrm{p} 32$, the site of the PCSK9 gene, in patients with autosomal dominant hypercholesterolemia. The Mutation enhanced the function of PCSK9 resulting in reduced LDLR expression and concomitant hypercholesterolemia [6]. Cohen reported a nonsense mutation with loss of function correlating with a $28 \%$ reduction in mean LDL-C levels. Clinical benefit was also reported with a reduced incidence of ischemic heart disease of $47 \%$ to $88 \%$ depending on the different sequence variations leading to loss of function mutations of PCSK9 [7]. Importantly this did not appear to affect other aspects of health. As our understanding of the interplay between PSCK9 and the LDLR has developed it has been an interesting discovery to find that statins produce PSCK9 up-regulation. Statins and ezetimibe decrease the amount of cholesterol in hepatocyte cells thereby stimulating LDLR up regulation. As a result this compensatory up regulation of PCSK9 may counteract the LDL-cholesterol lowering effectiveness of statins and ezetimibe [8]. As a result of these findings PSCK9 has become a novel target of clinical trials. These trials have

*Corresponding author: Alexander Dashwood, Unit 1001 Kirra Surf Apartments, 2 Creek Street, Kirra, 4225, QLD, Australia, Tel: 130074 4284; E-mail: icuris247@ googlemail.com

Received: November 24, 2014; Accepted: December 25, 2014; Published: January 01, 2015

Citation: Dashwood A, Jayasinghe R (2015) The Newest Frontier in Cholestero Management; PSCK 9. Cardiol Pharmacol 4: 127. doi:10.4172/2329-6607.1000127

Copyright: () 2015 Dashwood A et al. This is an open-access article distributed under the terms of the Creative Commons Attribution License, which permits unrestricted use, distribution, and reproduction in any medium, provided the original author and source are credited. 


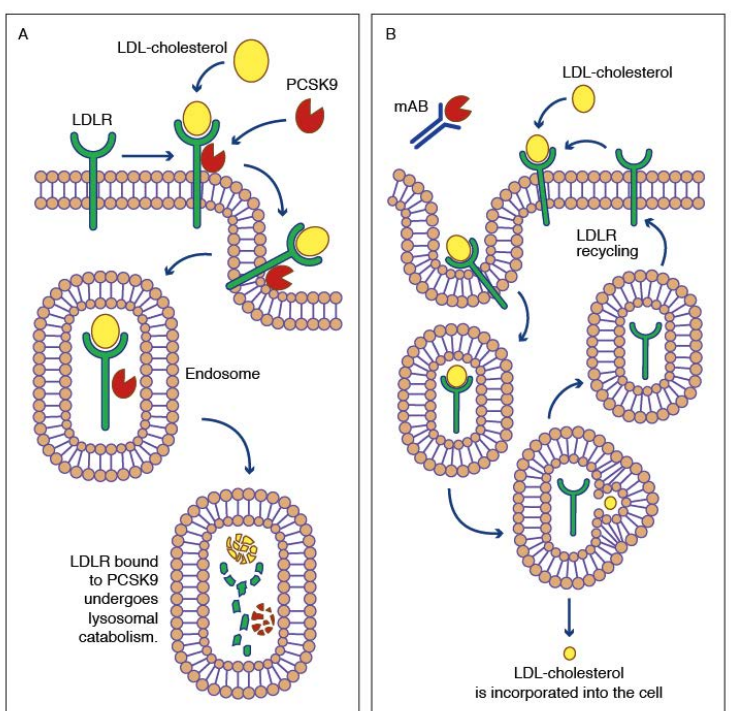

Figure 1: Proprotein convertase subtilisin/kexin type 9 (PCSK9) is primarily synthesized in the liver and is secreted in to the circulation where it binds to low-density lipoprotein receptor (LDLR). Interaction reduces Hepatocyte LDLR expression by driving internalization and subsequent lysosomal catabolism of the LDLR. b - Monoclonal Antibodies (mAbs) to PCSK9 block its interaction with the LDLR preventing its lysosomal destruction and promoting recycling of the receptor increasing LDLR express

aimed at exploring the efficacy of a monoclonal antibody targeting PCSK9, preventing LDLR degradation and improving circulating levels of LDL-C.

\section{Current Therapies and Clinical Trials}

\section{Evolocumab (AMG145)}

Evolocumab is a fully humanised monoclonal PCSK9 antibody administered subcutaneously. The initial phase 1 trials evaluated the effect of evolocumab on heterozygous familial hypercholesterolemia (FH) and non-FH patients who required statin therapy as well as healthy patients. Subcutaneous doses of 7, 21, 70, 140, 210 and 420 $\mathrm{mg}$ of evolucumab were used. Compared to placebo, evolocumab reduced LDL-C levels by $64 \%$ after 1 dose of $21 \mathrm{mg}$ or more. This improved to $81 \%$ with repeated dosing of $35 \mathrm{mg}$ or more [9]. Evolocumab was well tolerated with no serious adverse events. Subsequently the Goal Achievement after Utilizing an Anti PCSK9 Antibody in Statin Intolerant Subjects (GAUSS) trial was created to determine the efficacy of evolocumab in statin intolerant patients. 5 groups of patients who had a history of muscle related statin side effects received 12 weeks of evolocumab alone at 280,350 or $420 \mathrm{mg}$ or evolocumab $420 \mathrm{mg}$ plus ezetimibe $10 \mathrm{mg}$ or ezetimibe $10 \mathrm{mg}$ and placebo. Evolocumab and placebo was given subcutaneously at 4-week intervals. Treatment with evolocumab produced reductions in LDL-C levels from $41 \%$ to $63 \%$. Maximal effect was evident within 2 weeks of therapy and maintained throughout the 12 weeks of treatment. In the Evolocumab alone groups LDL-C levels of less than $2.5 \mathrm{mmol} / \mathrm{L}$ and less than $1.8 \mathrm{mmol} / \mathrm{L}$ were achieved in $54 \%$ and $18 \%$ respectively compared to ezetimibe alone which achieved $7 \%$ and $0 \%$ respectively. Importantly $94 \%$ of subjects completed the trial with favourable shortterm tolerability. Myalgia remained the most common reported side effect with 7 patients (7.4\%) taking evolocumab only and 6 patients (20\%) taking evolocumab/ezetimibe. Nasopharyngitis and fatigue were the other common reported effects [10]. The GAUSS-2 trial sought to evaluate evolocumab in patients of higher cardiovascular risk with at least 2 statin intolerances. It was a 12 week double blind study in which 307 patients were randomized to evolocumab, $140 \mathrm{mg}$ every two weeks (Q2W) or $420 \mathrm{mg}$ once a month (Q4W) or $10 \mathrm{mg}$ oral ezetimibe. Evolocumab reduced LDL-C from baseline by $53 \%$ to $56 \%$, corresponding to treatment differences versus ezetimibe of $37 \%$ to $39 \%$. $96 \%$ of patients completed treatment. Myalgia occurred in $18 \%$, $7 \%$ and $9 \%$ of patients receiving ezetimibe, evolocumab Q2W and evolocumab Q4W respectively [11].

The Phase II and III Monoclonal Antibody against PCSK9 to Reduce Elevated LDL-C in Subjects Currently Not Receiving Drug Therapy for Easy Lipid Levels (MENDEL) trials sought to establish the efficacy of evolocumab as a potential monotherapy in those not receiving statin therapy. The initial MENDEL trial reduced placebo corrected LDL-C levels by $37.3 \%$ to $52.5 \%$ from baseline. Patients had LDL-C levels between $2.5 \mathrm{mmol} / \mathrm{L}$ and $4.9 \mathrm{mmol} / \mathrm{L}$ and received 70 , $105,140 \mathrm{mg}$ of evolocumab or placebo Q2W or $280,350,420 \mathrm{mg}$ of evolocumab or placebo Q4W or $10 \mathrm{mg} /$ day of ezetimibe over 12 weeks. $120 \mathrm{mg}$ Q2W and $420 \mathrm{mg}$ Q4W produced the greatest effect with no evidence of dose limiting adverse effects [12]. Subsequently the Phase III MENDEL-2 trial conducted by Koren compared biweekly $140 \mathrm{mg}$ and monthly $420 \mathrm{mg}$ evolocumab, doses anticipated for clinical practise, with placebo and oral ezetimibe. 614 patients with fasting LDL-C levels of $\geq 2.5 \mathrm{mmol} / \mathrm{L}$ and $<4.9 \mathrm{mmol} / \mathrm{L}$ and a Framingham risk score of $\leq$ $10 \%$ were randomised. Evolocumab had a mean reduction in LDL-C from baseline of $55 \%$ to $57 \%$ more than placebo and $38 \%$ to $40 \%$ more than ezetimibe. $97 \%$ of patients completed the study with comparable muscle related side effects across all treatment groups at 4\% [13]. Most recently Blom completed a 52-week phase III trial evaluating the safety and efficacy of $420 \mathrm{mg}$ of evolocumab every 4 weeks. Depending on their ATP-III defined cardiovascular risk patients had a run in period of varying lipid-lowering regimes, ranging from diet alone to $80 \mathrm{mg}$ of atorvastatin and $10 \mathrm{mg}$ of ezetimibe. The overall mean reduction in LDL-C when compared to placebo was $57.0 \%$ Specifically, when compared to the run in therapy, evolocumab had a mean reduction in LDL-C of $55.7 \%$ in those who received diet control alone, $61.6 \%$ who took $10 \mathrm{mg}$ of atorvastatin, $56.8 \%$ amongst those who received $80 \mathrm{mg}$ of atorvastatin and $48.5 \%$ who received $80 \mathrm{mg}$ of atorvastatin and $10 \mathrm{mg}$ of ezetimibe. Additionally they found no decrement in efficacy from 12 weeks though to 52 weeks. Interestingly, unbound PSCK9 levels were measured at baseline and then compared at 13 and 37 weeks revealing a mean reduction of $91.1 \%$ and $86.9 \%$ respectively. Consistent with other studies the most common reported adverse events included nasopharyngitis, upper respiratory tract infections, influenza and back pain. Creatine kinase levels of five times above normal occurred in 7 patients $(1.2 \%)$ receiving evolocumab compared to 1 patient $(0.3 \%)$ in the placebo group [14]

\section{Alircumab (REGN727/SAR236553)}

Alircumab can be administered subcutaneously or intravenously. Initial phase 1 trials administered a single intravenous dose of up to 12 $\mathrm{mg} / \mathrm{kg}$ to 40 healthy subjects and $250 \mathrm{mg}$ of subcutaneous alircumab to 32 healthy patients. LDL-C was reduced by $65 \%$ with the intravenous administration showing greater efficacy. These trials were extended to patients with heterozygous $\mathrm{FH}$ with $\mathrm{LDL}-\mathrm{C} \geq 2.5 \mathrm{mmol} / \mathrm{L}$ receiving atorvastatin and non-FH with LDL-C $\geq 2.5 \mathrm{mmol} / \mathrm{L}$ and LDL-C $\geq$ $3.4 \mathrm{mmol} / \mathrm{L}$ who received atorvastatin or a modified diet respectively. Alircumab was administered subcutaneously on days 1,29, and 43 at doses of 50,100 , or $150 \mathrm{mg}$ with a reduction from baseline of $39.2 \%$, $53.7 \%$ and $61 \%$ respectively. The study size was small but showed 
promise for alircumab as potential monotherapy [15]. In a phase II placebo controlled trial, the efficacy of alircumab to lower LDL-C levels in primary hypercholesterolemia was compared to placebo. LDL-C levels at 12 weeks were reduced by $64.2 \%$ and $72.4 \%$ from baseline for $100 \mathrm{mg}$ and $150 \mathrm{mg}$ Q2W respectively whilst $200 \mathrm{mg}$ and $300 \mathrm{mg}$ Q $4 \mathrm{~W}$ produced $43.2 \%$ and $47.7 \%$ reductions respectively. The placebo group had a 5\% reduction [16]. Roth et al designed a study to measure the efficacy of alircumab when co-administered with $80 \mathrm{mg}$ or $10 \mathrm{mg}$ of atorvastatin, as compared with high-dose atorvastatin alone, in patients with LDL cholesterol levels of $\geq 2.5 \mathrm{mmol} / \mathrm{L}$. 92 patients had a run in period of $10 \mathrm{mg}$ of atorvastatin for at least 7 weeks. Subjects were randomly assigned to receive 8 weeks of treatment with $80 \mathrm{mg}$ of atorvastatin daily plus $150 \mathrm{mg}$ off subcutaneous alircumab Q2W, $10 \mathrm{mg}$ of atorvastatin daily plus alircumab Q2W, or $80 \mathrm{mg}$ of atorvastatin daily plus placebo Q2W. Reductions in LDL-C levels from baseline were $66.2 \%, 73.2 \%$ and $17.3 \%$ respectively for each group. Importantly all study drug group patients achieved LDL-C levels less than $2.5 \mathrm{mmol} / \mathrm{L}$. The study suggested that administration of alircumab with statins might benefit patients in whom LDL cholesterol has not been reduced to recommended levels. There was also no significant difference between $80 \mathrm{mg}$ or $10 \mathrm{mg}$ atorvastatin plus the study drug. This suggested a limit to the up-regulation of LDL-receptor activity and that effects of alircumab are not synergistic when added to statins [17].

\section{RN316}

RN316 is a humanized PCSK9 monoclonal antibody administered intravenously that has recently been tested for its efficacy and safety in clinical trials. In the initial phase I studies, subjects with primary hypercholesterolemia were administered multiple doses of RN316 (0.25-1.5 mg/kg/week) for a period of 4 weeks. LDL-C levels were reduced by up to $90 \%$ from baseline with additional improvements in triglyceride and high-density lipoprotein cholesterol (HDL-C) concentrations. Gumbiner et al followed this up with a Phase II trial where hypercholesterolemic subjects receiving high dose statins were assigned to RN316 (dose range: $0.25-6.0 \mathrm{mg} / \mathrm{kg}$; IV) every 4 weeks for a period of 12 weeks. 90 participants with an LDL-C $\geq 2.5 \mathrm{mmol} / \mathrm{L}$ were given the study drug or placebo while on concurrent high doses of atorvastatin ( 40 or $80 \mathrm{mg}$ ), rosuvastatin $(20$ or $40 \mathrm{mg}$ ), or simvastatin ( 40 or $80 \mathrm{mg}$ ). In the second arm 45 subjects with LDL-C $\geq 2.0 \mathrm{mmol} / \mathrm{L}$ was administered study drug or placebo while on atorvastatin $80 \mathrm{mg}$ or rosuvastatin $40 \mathrm{mg}$. After 12 weeks the pooled mean reduction in LDL-C for those receiving the study drug was $56 \%$ compared with $4 \%$ in the placebo group. Again HDL-C concentrations increased but triglycerides showed no significant improvement. RN316 was well tolerated with no study drug related adverse events [18]. Currently a large multicentre phase III trial aims to recruit 6300 subjects with the aim to evaluate the efficacy, safety and tolerability of RN316 compared to placebo. Its primary objective is to demonstrate the superior efficacy to reduce major cardiovascular events in subjects with high risk of major cardiovascular events who are on maximally tolerated lipidlowering therapy but have an LDL-C $\geq 2.5 \mathrm{mmol} / \mathrm{L}$ or non-HDL-C $\geq$ $3.4 \mathrm{mmol} / \mathrm{L}$.

\section{Safety issues related to PCSK9 inhibition}

Whilst the discussed trials have shown encouraging results for pharmacological inhibition of PCSK9, the long-term effects upon human health remain unknown. Mice animal models lacking PCSK9 develop normally and have no gross neurological defects [19]. However, inactivation of PCSK9 in embryos of zebra fish results in disordered neuronal development and death [20]. Encouragingly we have identified individuals who are heterozygous for loss-of-function mutations in PCSK9 and appear to be healthy with a normal lifespan [21]. Zhao et al. identified a healthy fertile college graduate who was a compound heterozygote for two inactivating mutations in PCSK9 who had a strikingly low plasma level of LDL-C $(0.4 \mathrm{mmol} / \mathrm{L})$. Recently Jasmin SB found that at autopsy, individuals with Alzheimer's disease had a 2-fold increased expression of PCSK9. Its role remains unclear but it may play a role in the homeostatic regulation of neuronal repair [22].

\section{Discussion}

There has been a rapid progress in our understanding of PCSK9 and its role in LDL metabolism. It provides a clear example of our current technological abilities in translational sciences to produce novel therapeutic targets. Monoclonal antibody therapy has been shown to achieve a reduction in LDL-C levels of $50 \%$ to $75 \%$. Whilst it is unlikely these monoclonal antibodies will replace HMG-CoA reductase inhibitors in the near future due to production costs, health care economics and mode of delivery they are likely to provide a robust and reasonable alternative for certain populations; those intolerant to statins, those who do not attain recommended LDL-C levels despite maximal therapy and finally individuals with FH. In the context of the American College of Cardiology/American Heart Association [23] guidelines, PCSK9 monoclonal antibodies provide a realistic alternative, however long-term antibody use remains unevaluated.

\section{References}

1. National cholesterol education programme (NCEP) (2002) Third Report of the National Cholesterol Education Program (NCEP) Expert Panel on Detection, Evaluation, and Treatment of High Blood Cholesterol in Adults (Adult Treatment Panel III) final report. Circulation 106: 3143-3421.

2. Rosenson RS (2003) Rosuvastatin: a new inhibitor of HMG-coA reductase for the treatment of dyslipidemia. Expert Rev Cardiovasc Ther 1: 495-505.

3. Baigent C, Keech A, Kearney PM, Blackwell L, Buck G, et al. (2005) cholestero trialists (CTT) collaborators. Efficacy and safety of cholesterol-lowering treatment: prospective meta-analysis of data from 90.056 participants in 14 randomized trials of statins. Lancet 366: 1267-1278.

4. Horton JD, Cohen JC (2007) Molecular biology of PCSK9: its role in LDL metabolism. Trends Biochem Sci 32: 71-77.

5. Michael HD (2013) Emerging low-density lipoprotein therapies: targeting PCSK9 for low density lipoprotein reduction journal of clinical lipidology 7: 1115.

6. Abifadel M, Varret M, Rabès JP, Allard D, Ouguerram K, et al. (2003) Mutations in PCSK9 cause autosomal dominant hypercholesterolaemia. Nat Genet 34: 154-156.

7. Cohen JC, Boerwinkle E, Mosley TH Jr, Hobbs HH (2006) Sequence variations in PSCK9, low LDL, and protection against coronary heart disease. $\mathrm{N}$ Eng $\mathrm{J}$ Med 354: 1264-1272.

8. Dubuc G, Chamberland A, Wassef H, Davignon J, Seidah NG, et al. (2004) statins upregulate PCSK9, the gene encoding the proprotein convertase neural apoptosis regulated convertase-1 implicated in familial hypercholesterolemia. Arterioscler Thromb Vasc Biol 24: 1454-1459.

9. Dias CS, Shaywitz AJ, Wasserman SM, Smith BP, Gao B, et al. (2012) EffectsofAMG145 on low-density lipoprotein cholesterol levels: results from 2 randomized, double-blind, placebo- controlled, ascending-dose phase 1 studies in healthy volunteers and hypercho- lesterolemic subjects on statins. J Am Coll Cardiol 60: 1888-1898.

10. Sullivan D, Olsson AG, Scott R, Kim JB, Xue A, et al. (2012) Effect of monoclonal antibody to PCSK9 on low-density lipoprotein cholesterol levels in statin-intolerant patients: the GAUSS randomized trial. JAMA 308: 2497-2506.

11. Stroes E, Colquhoun D, Sullivan D, Civeira F, Rosenson RS, et al. (2014) Anti-PCSK9 Antibody Effectively Lowers Cholesterol in Patients With Statin Intolerance The GAUSS-2 Randomized, Placebo-Controlled Phase 3 Clinical Trial of Evolocumab. J Am Coll Cardiol 63: 2541-2548.

12. Koren MJ, Scott R, Kim JB, Knusel B, Liu T, et al. (2012) Efficacy, safety, and 
Citation: Dashwood A, Jayasinghe R (2015) The Newest Frontier in Cholesterol Management; PSCK 9. Cardiol Pharmacol 4: 127. doi:10.4172/23296607.1000127

tolerability of a mono- clonal antibody to proprotein convertase subtilisin/kexin type 9 as mono- therapy in patients with hypercholesterolaemia (MENDEL): a randomised, double-blind, placebo-controlled, phase 2 study. Lancet 380:1995-2006.

13. Koren MJ, Lundqvist $P$, Bolognese $M$, Neutel JM, Monsalvo ML, et al. (2014) Anti-PCSK9 Monotherapy for Hypercholesterolemia. The MENDEL-2 Randomized, Controlled Phase III Clinical Trial of Evolocumab. J Am Coll Cardiol 63: 2531-2540.

14.

15. Blom DJ, Hala T, Bolognese M, Michael JL, Phillip DToth, et al. (2014) A 52Week Placebo-Controlled Trial of Evolocumab in Hyperlipidemia. N Engl J Med 370:1809-1819.

16. Stein EA, Mellis S, Yancopoulos GD, Neil S, Douglas L, et al. (2012) Effect of a monoclonal antibody to PCSK9 on LDL cholesterol. N Engl J Med 366: 1108-1118.

17. McKenney JM, Koren MJ, Kereiakes DJ, Hanotin C, Ferrand AC, et al. (2012) Safety and efficacy of a mono- clonal antibody to proprotein convertase subtilisin/kexin type 9 serine protease, SAR236553/REGN727, in patients with primary hypercholesterolemia receiving ongoing stable atorvastatin therapy. $J$ Am Coll Cardiol 59: 2344-2353.
18. Roth EM, McKenney JM, Hanotin C, Gaelle A, Evan A et al.(2012) Atorvastatin with or without an antibody to PCSK9 in primary hypercholesterolemia. N Engl J Med367:1891-1900.

19. Gumbiner B, Joh T, Udata C (2012) Effects of 12 weeks of treatment with RN316 (PF-04950615), a humanized IgG2 $\Delta$ a monoclonal antibody binding proprotein convertase subtilisin kexin type 9 , in hypercholesterolemic subjects on high and maximal dose statins. Circulation 126: 2782.

20. Shirya R, David EC, Rita G, Norma NA, Yuriy B, et al. (2005) Decreased plasma cholesterol and hypersensitivity to statins in mice lacking Pcsk9. Proc Natl Acad Sci 102:5374-5379.

21. Poirier S, Prat A, Marcinkiewicz E, Paquin J, Chitramuthu BP et al. (2006) Implication of the proprotein convertase NARC-1/PCSK9 in the development of the nervous system. J Neurochem 98: 838-850.

22. Cohen JC, Boerwinkle E, Mosley TH Jr, Hobbs HH (2006) Sequence variations in PCSK9, low LDL, and protection against coronary heart disease. N Engl J Med 354:1264-1272.

23. Jasmin SB (2012) A putative role for PCSK9 in synaptic remodeling and plasticity in response to brain injury: implications for Alzheimer's disease.

24. Stone N, Robinson J, AH L (2013) ACC/AHA guideline on the treatment of blood cholesterol to reduce atherosclerotic cardiovascular risk in adults: a report of the American College of Cardiolssssogy. 